\title{
Intraoperative Impedance Measurements for the Evaluation of Perimodiolar Location of Cochlear Implant Electrode - Own Experience
}

\author{
O. StieleR ${ }^{a, b, *}$, M. KARLiK ${ }^{c}$ And W. SzYfTER ${ }^{b}$ \\ ${ }^{a}$ Institute of Acoustics, Adam Mickiewicz University, Poznań, Poland \\ ${ }^{b}$ Department of Otolaryngology, Poznań University of Medical Sciences \\ Przybyszewskiego 49, 60-355 Poznań, Poland
}

\begin{abstract}
${ }^{c}$ Department of Phoniatrics and Audiology, Poznań University of Medical Sciences, Poznań, Poland
Evaluation of insertion of a cochlear implant electrode into the cochlea includes radiological techniques: plain X-ray of the skull, cochlear projection (based on Stenvers projection), computer tomography. These methods allow generally postoperative evaluation. During surgery the insertion of the electrode is assessed based on anatomical structures and markers placed on the electrode. The measurement of the electrode impedance is also an important tool. 60 patients implanted with the Contour cochlear implant with pre-shaped electrode were examined. Age o patients: mediana 4.25 (1.5-54.5). Examination includes intraoperative impedance measurement performed in two stages: (1) after the electrode insertion, and (2) after removal of the stylet. Measurements were done in Common Ground (CG) and Monopolar 1 (MP1) stimulation modes. Measurements recorded in two modes and in 3 different parts of the cochlea (apical, middle, basal) were compared. The average difference value (before-after) was for the CG mode: $0.45 \mathrm{k} \Omega$, and for the MP1 mode: $0.39 \mathrm{k} \Omega$. For the CG mode the difference values were: 0.64, 0.44 and 0.28 toward apical part. For the MP1 mode the difference values were: $0.47,0.43$ and 0.27 toward apical part of the cochlea.
\end{abstract}

PACS numbers: 43.90.+v, 43.58.-e, 43.60.-c, 43.60.+d

\section{Introduction}

As physical and medical knowledge developed it became to restore auditory-verbal communication in patients with a bilateral deafness and in persons with a profound bilateral perceptive hearing loss. Today such communication can be restored with the help of a cochlear implant inserted into the internal ear. This operation is performed on patients, in whom cochlear hair cells, which are responsible for the transmission of the signal to the hearing nerve, have been damaged. Patients suffering from this dysfunction cannot benefit from the conventional hearing aids as the acoustic compensation of the deficit does not stimulate the higher parts of the hearing pathway. This condition is caused by the lack or only residual communication of the peripheral hearing system with the hearing nerve. A wide range of perceptive hearing loss can be compensated by classical hearing aids. A profound damage of the inner hair cells results in permanent hearing loss. In pure tone audiometry examination this pathology is shown as curves of air conduction lowered from 90 to $120 \mathrm{~dB}$ HL.

A cochlear implant is an electronic device, which replaces the activity of the damaged cochlea. The basic function of the cochlear implant is to bypass the receptor and provide the information carried by the speech

* corresponding author; e-mail: osaku@umed.poznan.pl signal, analysed in the processor, in the form of electrical stimuli, directly into the ends of the hearing nerve. The cochlear implant system consists of two parts, the inner part, which is inserted surgically, and the outer part - the speech processor. The inner part of the Nucleus implant comprises an internal coil, a magnet and a transducer. The functions of decoder and the simulation control system are performed by a hybrid integrated circuit, placed in a titanium coated ceramic casing. The part inserted into the tympanic canal consists of a 22-channel electrode. Each channel is made of platinum and insulated with an elastic silicone. The reference electrodes in Nucleus 24 implant include a ball electrode placed under the temporal muscle (MP1) and an electrode on the implant's base (MP2). The electrodes outside the cochlea are reference electrodes, permitting application of different ways of electrical stimulation (a different area of the electrical field).

A contour electrode has a special stylet, which makes it easier to insert it. When the electrode has been inserted into the cochlea the stylet is removed and the electrode is curled up and approaches the modiolus. This solution is intended to reduce the range of the electrical field that is generated and in this way makes the electrical stimulation more precise and reduces the consumption of electrical energy needed to cause a sound sensation.

Band filtration, signal analysis and conversion of the acoustic signal into an electrical code are made in the speech processor. In this way the acoustic signal is con- 
verted into an electrical signal - physiologically this process is the basic activity of the hearing receptor. The signal is sent to the electrode, to which a set frequency range of the input signal is assigned. This process is managed by the frequency allocation table (FAT), defined in the speech processor parameters. The analysed signal is within the range of about 200 and $8000 \mathrm{~Hz}$, which is divided into bands the number of which corresponds to the number of active electrodes - usually from 20 to 22 . The number of active electrodes depends on the number of electrodes inserted into the cochlea, which is influenced by the anatomic conditions present during the operation procedure and also by the speech coding strategy applied.

The acoustic pressure level of the signal is proportional to the impulse pitch $[\mu \mathrm{A}]$ and its width $[\mu \mathrm{s}]$, within the limits of stimulation thresholds set in the speech processor: $\mathrm{T}$ - threshold level, $\mathrm{C}$ - comfort level $[\mu \mathrm{A}]$.

The insertion of the cochlear implant's electrode into the cochlea can be evaluated by means of radiological techniques - an image of the skull (front-back projection), cochlear projection (based on Stenvers projection), and computer tomography. These methods permit evaluation in the post-operative period. During the operation the insertion of the electrode is assessed by the otosurgeon, based on the visible anatomical structures and relevant markers on the implant [1].

Advancements of knowledge about physics and electrophysiology permitted direct application of telemetric techniques. Cochlear implants used presently permit bi-directional communication between the inner and outer parts of the device. In practice this means that both stimulation and reading of the response of the physiological system to electrical stimuli transmitted via the implant are possible. Measurement of the electrode impedance is an important evaluation tool. Measurement of the electrode impedance defines the electrical resistance in the place of contact between the electrode and the tissue. In this way it is possible to determine which electrodes should be excluded from stimulation as well as how the stimulation level should be adjusted because of voltage compliance. During the measurement electrodes are stimulated with the energy of 100 units (Current Level CL). The 1-225 scale refers to the intensity range of the electrical current from about $10 \mathrm{~mA}$ to about $1750 \mathrm{~mA}$. The scale unit corresponds to a $2 \%$ amplitude change (logarithmic scale) [2-4].

Measurements of impedance made during the operation help inform the operator about the registered electrical resistance. When this information is analysed together with the anatomic conditions, a decision about subsequent procedure can be made (e.g. the electrode should be inserted more deeply or the electrode should be moved slightly back).

\section{Aim}

Evaluation of the intraoperative measurement of electrode impedance in patients with Contour electrode cochlear implant.

\section{Material and methods}

60 patients, aged between $1.5-54.5$ years (median 4.25 ) with a Nucleus implant with Contour electrode of a pre-defined shape were analysed.

The electrodes were inserted in all the patients by the same surgeon. The examined group included patients with a normal cochlea in the pre-operative radiological scan (computed tomography) and correct anatomic structures found during the operative procedure.

Intraoperative measurements of impedance were made at two stages: (1) directly after the insertion of the electrode into the cochlea and (2) after the removal of the stylet. The measurements of impedance were made in the Common Ground (CG) and Monopolar 1 (MP1) modes. In the CG mode impedance is measured between the electrodes inserted into the cochlea. In the MP1 mode impedance is measured between each of the 22 electrodes inserted into the cochlea and the ball electrode placed under the temporal muscle. Measurement of impedance in the MP2 mode at this stage of the procedure depends on the operative conditions as it requires covering of the contact of MP2 electrode with the tissues, which is in the transducer. The measurement principle in each of the simulation modes is presented in Fig. 1. Impedance recorded in both measurements was compared.

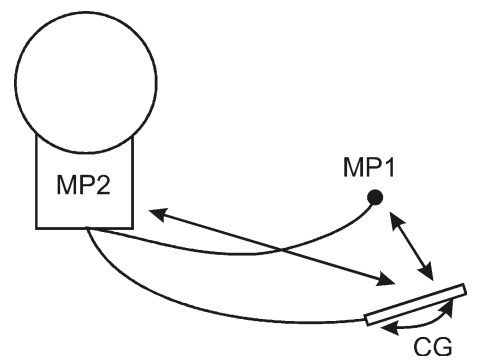

Fig. 1. Cochlear implant scheme with modes of stimulation.

The following equipment was used in the measurements:

- PC with Windows XP Professional,

- Implant Portable Programming System (PPS) for Nucleus Contour implants, Pod programmer for Freedom Contour Advanced implants,

- Nucleus Freedom, Sprint speech processor

- Cochlear made CustomSound, CustomSound EP software.

The measurement process was computer controlled. Implant software and communication interfaces were used. 


\section{Results}

Mean impedance values in $[\mathrm{k} \Omega]$ in the $\mathrm{CG}$ mode and in the MP1 mode before and after the removal of the stylet for individual electrodes and mean values of the difference are presented in Table Ia,b.

TABLE Ia

Mean impedance values in $[\mathrm{k} \Omega]$ in the $\mathrm{CG}$ mode.

\begin{tabular}{l|c|c|c|c|c|c|c|c}
\hline \hline $\begin{array}{l}\text { No. of } \\
\text { electr. }\end{array}$ & 22 & 21 & 20 & 19 & 18 & 17 & 16 & 15 \\
\hline before & 11.32 & 11.31 & 10.75 & 10.98 & 9.79 & 11.22 & 10.14 & 10.17 \\
after & 10.99 & 10.96 & 10.39 & 12.16 & 9.57 & 9.84 & 9.70 & 10.32 \\
differ. & 0.33 & 0.35 & 0.36 & -1.18 & 0.22 & 1.38 & 0.44 & -0.15 \\
\hline \hline $\begin{array}{l}\text { No. of } \\
\text { electr. }\end{array}$ & 14 & 13 & 12 & 11 & 10 & 9 & 8 & 7 \\
\hline before & 10.37 & 10.21 & 9.93 & 9.74 & 9.44 & 9.66 & 9.20 & 9.29 \\
after & 10.08 & 9.70 & 9.12 & 9.25 & 9.09 & 9.10 & 8.63 & 8.85 \\
differ. & 0.29 & 0.51 & 0.81 & 0.49 & 0.35 & 0.56 & 0.57 & 0.44 \\
\hline \hline $\begin{array}{l}\text { No. of } \\
\text { electr. }\end{array}$ & 6 & 5 & 4 & 3 & 2 & 1 & & \\
\hline before & 9.56 & 10.09 & 10.29 & 10.57 & 10.65 & 11.24 & & \\
after & 9.10 & 9.67 & 9.67 & 9.94 & 10.28 & 10.89 & & \\
differ. & 0.46 & 0.42 & 0.62 & 0.63 & 0.37 & 0.35 & &
\end{tabular}

TABLE Ib

Mean difference of impedance value in the MP1 mode.

\begin{tabular}{|c|c|c|c|c|c|c|c|c|}
\hline $\begin{array}{l}\text { No. of } \\
\text { electr. }\end{array}$ & 22 & 21 & 20 & 19 & 18 & 17 & 16 & 15 \\
\hline before & 13.97 & 13.90 & 13.32 & 13.33 & 12.23 & 12.55 & 12.50 & 12.39 \\
\hline after & 13.84 & 13.52 & 12.76 & 13.15 & 12.02 & 12.34 & 12.21 & 12.21 \\
\hline differ. & 0.13 & 0.39 & 0.56 & 0.18 & 0.22 & 0.21 & 0.29 & 0.18 \\
\hline $\begin{array}{l}\text { No. of } \\
\text { electr. }\end{array}$ & 14 & 13 & 12 & 11 & 10 & 9 & 8 & 7 \\
\hline before & 12.81 & 12.43 & 12.15 & 11.97 & 11.84 & 11.97 & 11.34 & 11.62 \\
\hline after & 12.71 & 12.11 & 11.57 & 11.47 & 11.33 & 11.28 & 10.75 & 10.86 \\
\hline differ. & 0.10 & 0.32 & 0.58 & 0.50 & 0.52 & 0.69 & 0.60 & 0.76 \\
\hline $\begin{array}{l}\text { No. of } \\
\text { electr. }\end{array}$ & 6 & 5 & 4 & 3 & 2 & 1 & & \\
\hline before & 11.85 & 12.21 & 12.45 & 12.59 & 12.24 & 13.04 & & \\
\hline after & 11.22 & 11.70 & 11.80 & 11.92 & 11.80 & 12.22 & & \\
\hline differ. & 0.63 & 0.51 & 0.65 & 0.67 & 0.44 & 0.82 & & \\
\hline
\end{tabular}

Mean values of the impedance difference in the individual stimulation modes before and after the removal of the stylet are shown in Fig. 2.

The mean difference of the impedance value for the electrodes before and after the removal of the stylet for the $\mathrm{CG}$ mode was $0.45 \mathrm{k} \Omega$ and for the MP1 mode was $0.39 \mathrm{k} \Omega$.

In order to evaluate the difference in the individual parts of the cochlea, electrodes were divided into three ranges: (A) peak - electrodes 22 to 16, (B) middle electrodes 15-8, and (C) basic - electrodes 7-1. Mean values of the impedance differences between the measurements for the individual parts of the cochlea are presented in Table II.

The authors ascribe the lower mean value of the impedance difference after the removal of the stylet in the

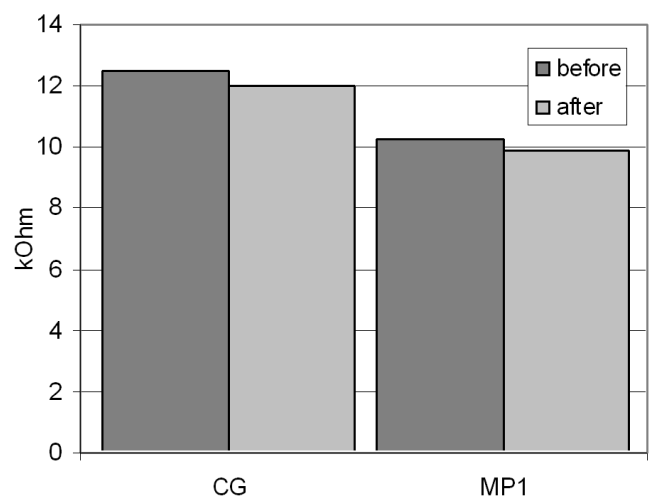

Fig. 2. Cochlear implant scheme with modes of stimulation.

TABLE II

Mean values of the impedance for the individual parts of the cochlea.

\begin{tabular}{l|c|c|c}
\hline \hline electrode & $22-16$ & $15-8$ & $7-1$ \\
\hline CG before & 13.11 & 12.11 & 12.29 \\
CG after & 12.83 & 11.68 & 11.65 \\
CG difference & 0.28 & 0.44 & 0.64 \\
\hline MP1 before & 10.79 & 9.84 & 10.24 \\
MP1 after & 10.52 & 9.41 & 9.77 \\
MP1 difference & 0.27 & 0.43 & 0.47
\end{tabular}

MP1 mode to the position of the ball electrode, which is relatively far from the cochlear structures.

\section{Conclusions}

Based on the results of the measurements made during the implantation operation it was found that after the removal of the stylet in the Contour electrode impedance was lowered. It confirms the change of the electrode's shape after the stylet removal. The electrode approaches the modiolus and the distance between the electrode and the modiolus is shorter. This was observed mainly in the basic and middle part of the cochlea. The procedure of the stylet removal influence mainly electrodes responsible for middle and high frequency sounds. In subsequent research it is planned to increase the number of patients examined and expand the measurements to include the results recorded during the connection of the speech processor, i.e. about $4-6$ weeks after the operation.

\section{References}

[1] W. Szyfter, A. Pruszewicz, M. Karlik, M. Kawczyński, A. Sekula, P. Świdziński, M. Magierska, Eur. Arch. Oto-Rhino-Laryngol. 260, 460 (2003).

[2] Nucleus Technical Reference Manual, Cochlear Corporation, Englewood, 1999.

[3] O. Stieler, Pol. J. Envir. Stud. 15, 103 (2006).

[4] O. Stieler, M. Karlik, Arch. Acous. 30, 265 (2005). 